\title{
Regeneration potential of adult cardiac myocytes
}

\author{
Cell Research (2013) 23:978-979. doi:10.1038/cr.2013.78; published online 18 June 2013
}

Although adult cardiac myocytes (CMs) have very little proliferative potential, fetal CMs divide robustly. The mechanisms underlying the post-mitotic state of CMs are poorly understood; however, recently Mahmoud et al. identified a homeodomain transcription factor, Meis1, which controls postnatal $\mathrm{CM}$ cell cycle.

In mammals, cardiac growth during embryonic development is primarily dependent on hyperplasia (increase in cell number). Cardiac myocytes (CMs), however, lose their ability to proliferate soon after birth and heart growth switches predominantly to hypertrophy (an enlargement of cell size). The proliferative capacity of postnatal CMs has been actively debated in the past few years. However, there is growing consensus that $\mathrm{CMs}$ renew continuously throughout life in mammalian hearts but at a very low rate [1]. Estimates of this renewal rate range from $0.5 \%-1 \%$ per year. The origin of new CMs has been controversial as well; whether they arise from differentiation of resident cardiac stem cells/cardiac progenitors or from proliferation of pre-existing CMs. Senyo et al. [2] recently addressed this question using a combined approach of genetic tracking and non-radioactive stable nitrogen isotope ${ }^{15} \mathrm{~N}$-labeled DNA imaging in mice. They demonstrated that $\mathrm{CM}$ renewal occurs at a very low rate $(0.8 \%$ per year) in adult mice and that most of the new CMs are generated from pre-existing $\mathrm{CMs}$ rather than from progenitor cells.

Thus, it has been believed for some time that the postnatal mammalian heart has no regeneration potential to compensate for lost CMs after injury.
In contrast, many lower vertebrates, such as amphibia or fish, are able to effectively regenerate their hearts. The zebrafish heart can completely regenerate after $20 \%$ of the ventricle is amputated. Genetic lineage-tracing methods showed that regenerated zebrafish CMs are derived from proliferation of preexisting CMs, not endogenous progenitor or stem cells [3]. While mice retain this regenerative capacity for the first week after birth, it is then permanently lost. This regenerative response, similar to zebrafish, is mediated by pre-existing CM proliferation [4]. Clearly, endogenous regeneration in adult heart is not nearly sufficient to compensate for lost myocardium after infarction, but very limited adult $\mathrm{CM}$ proliferation at the border of the infarct zone has recently been demonstrated [2]. Thus, reactivating the $\mathrm{CM}$ proliferative potential might be a feasible strategy to regenerate the injured adult heart if the mechanisms underlying CM cell cycle exit are better understood.

The molecular mechanisms that underlie the inability of adult CMs to reenter the cell cycle and divide are under intense investigation. Recent reports suggest that there are at least three different levels of regulations limiting CM proliferation: 1) pre-transcriptional heterochromatin-mediated gene silencing of positive cell cycle regulators [5], 2) post-transcriptional regulation through microRNAs (miRNAs) [6], and now 3) transcriptional activation of negative cell cycle regulators [7] (Figure 1).

The first report of successfully reversing the post-mitotic state of adult CMs demonstrated the importance of epigenetic regulation of cell cycle gene silencing in adult CMs [5]. Similar to senescent cells, the gene-silencing epigenetic histone mark $\mathrm{H} 3 \mathrm{~K} 9$ me 3 becomes enriched on promoters of cell cycle progression genes ( $c d c 2$ and $P l k 1$ ) during the differentiation from fetal to adult CMs. H3K9me3 is identified by a histone reader protein, heterochromatin protein 1 (HP1), which is recruited by RB to cell cycle progression gene promoters and packages these genes into stably silenced heterochromatic foci. Deleting RB and p130 disrupted heterochromatin formation, resulting in reactivation of cell cycle progression genes. In addition, investigators identified a microRNA family, miR-15, which can regulate cell cycle exit of neonatal CMs [6]. Inhibition of miR-15 from birth to young adulthood by injection of locked nucleic acid-modified miRNAs did not affect initial infarct size or systolic function. These miR-15-inhibited hearts, however, demonstrated induction of $\mathrm{CM}$ proliferation and a gradual improvement in left systolic function after infarction. Subsequently, a number of miRNAs capable of stimulating $\mathrm{CM}$ proliferation were identified using high-throughput screening of the human genome miRNA library [8]. Of these miRNAs, viral delivery of two miRNAs (has-miR-590 and has-miR199a) promoted CM proliferation in neonatal hearts and induced cell cycle reentry in adult hearts. The targets of these miRNAs remain to be determined. These findings indicate that adult CM cell cycle can be manipulated, which may be a potential tool to induce $\mathrm{CM}$ regeneration.

Recently, Mahmoud et al. [7] iden- 
A

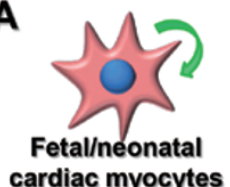

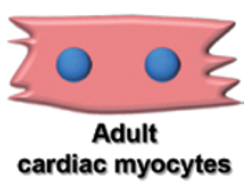

cardiac myocytes
B 1) Pre-transcriptional silencing

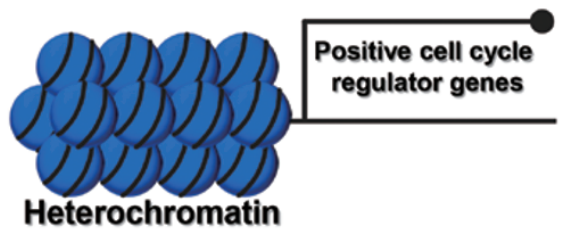

2) Post-transcriptional regulation
3) Transcription activation

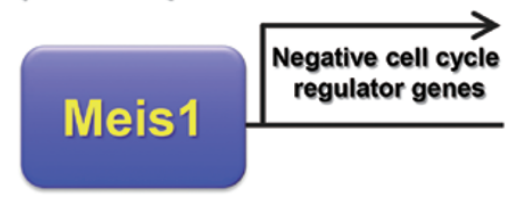

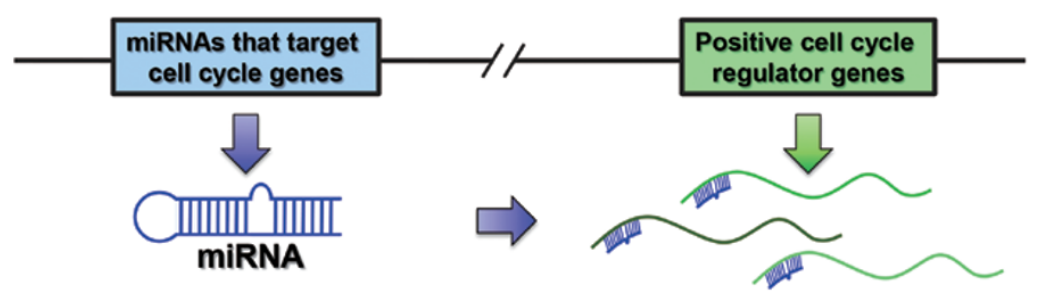

Figure 1 Mechanisms that limit regenerative capacity of postnatal CMs. Three mechanisms have been identified to explain the limited cell cycle progression in adult CMs. 1) Pre-transcriptional heterochromatin-mediated gene silencing: positive cell cycle regulator genes are marked by silencing epigenetic marks (H3K9me3, HP1s) and packed into heterochromatin, resulting in permanent silencing. 2) miRNA regulation: miRNAs post-transcriptionally suppress gene clusters involved in CM regeneration. 3) Transcriptional activation: Meis1 activates transcription of negative cell cycle regulator genes.

tified a transcription factor, Meis 1 , that regulates cell cycle exit and regeneration capacity of neonatal CMs. Meis1 belongs to the TALE (three amino acid loop extension) family of homeodomain proteins, which are required for normal haematopoiesis and heart development. The authors demonstrated that deleting Meis1 specifically in CMs extended the postnatal proliferative window of CMs from 7 to 14 days. In addition, specifically deleting Meis 1 in adult CMs resulted in cell cycle reentry and induction of $\mathrm{CM}$ proliferation in adult $\mathrm{CMs}$ without inducing CM hypertrophy or reducing heart function. In contrast, postnatal overexpression of Meis1 reduced neonatal $\mathrm{CM}$ proliferation and inhibited the heart regeneration response that is usually observed in the first week after birth. Meis1 deletion resulted in downregulation of cyclin-dependent kinase inhibitors including Ink family members (p15, p16 and p19) and Cip/Kip family members (p21 and p57), which are usually upregulated in the postnatal stage. Ink and Cip/Kip family gene promoters contain consensus Meis1-binding sequences, suggesting that Meis1 directly regulates these genes. Thus, the authors concluded that Meis 1 promotes $\mathrm{CM}$ cell cycle exit and reduces regeneration potential by activating negative cell cycle regulator genes. Over 400 potential target genes of Meis1 have been identified in embryonic CM development [9]. Further investigation will be required to identify additional direct targets of
Meis1 that orchestrate cell cycle exit in neonates and to study whether transient manipulation of Meis 1 can induce heart regeneration in adult hearts after injury.

Over the past decade, there has been intense interest in developing a clinically viable strategy to regenerate myocardium after injury. Most of these efforts have focused on stem cells and cardiac cell transplantation. However, the study on Meis1 [7] highlights a novel and promising strategy, namely, stimulating pre-existing CM regeneration. Studies that further our understanding of the mechanisms of limiting CM renewal will bring us closer to that goal.

Kyohei Oyama ${ }^{1}$, Danny El-Nachef ${ }^{1}$, W Robb MacLellan ${ }^{1}$

${ }^{1}$ Departments of Medicine and Cardiology, Center for Cardiovascular Biology, Institute for Stem Cell Research, University of Washington School of Medicine, Seattle, WA 98195, USA Correspondence: W Robb MacLellan Tel: +1-206-543-8584; Fax: +1-206-616-4847 E-mail: WRMacLellan@cardiology.washington. edu

\section{References}

1 Laflamme MA, Murry CE. Nature 2011; 473:326-335.

2 Senyo SE, Steinhauser ML, Pizzimenti CL, et al. Nature 2013; 493:433-436.

3 Jopling C, Sleep E, Raya M, et al. Nature 2010; 464:606-609.

4 Porrello ER, Mahmoud AI, Simpson E, et al. Science 2011; 331:1078-1080.

5 Sdek P, Zhao P, Wang Y, et al. J Cell Biol 2011; 194:407-423.

6 Porrello ER, Mahmoud AI, Simpson E, et al. Proc Natl Acad Sci USA 2013; 110:187-192.

7 Mahmoud AI, Kocabas F, Muralidhar SA, et al. Nature 2013; 497:249-253.

8 Eulalio A, Mano M, Dal FM, et al. Nature 2012; 492:376-381.

9 Wamstad JA, Alexander JM, Truty RM, et al. Cell 2012; 151:206-220. 